\title{
Fuentes y fases de redacción en el libro XXXI de Amiano Marcelino*
}

\author{
Álvaro Sánchez-Ostiz \\ Universidad de Navarra \\ asostiz@unav.es \\ ORCID iD: https://orcid.org/0000-0002-6925-9016
}

Sources and phases of composition in Ammianus XXXI

\begin{abstract}
El artículo propone que algunas de las discontinuidades estructurales en el último libro de Amiano Marcelino se explican porque pertenecen a secciones que fueron añadidas al núcleo original del relato en una fase posterior de redacción y, por lo tanto, no dependen de la fuente principal: la digresión sobre los hunos y los alanos en XXXI 2, secciones concretas dentro de la narrativa militar (XXXI 6.7-8, 8.7-8, 15.10 y 13) y la nota fisonómica al final de la necrología de Valente. Estas secciones no sólo no están perfectamente integradas en el flujo narrativo, sino que además añaden contenidos discordantes con los argumentos principales en el libro XXXI. Todo ello permite profundizar en la comprensión de la técnica literaria del historiador y de las aparentes incongruencias del último libro de la obra de Amiano.
\end{abstract}

Palabras clave: Amiano Marcelino; libro XXXI; fuentes, estructura y composición de las Res Gestae; excursos; narrativa militar; intertextualidad; fisonomía; caracterización de los personajes.
This paper proposes that some structural discontinuities in the latest book by Ammianus can be explained because those sections were added to a first version of the text in a subsequent phase of composition and therefore do not depend on the main source: the digression on the Huns and Alans in XXXI 2, some sections of military narrative (XXXI 6.7-8, 8.7-8, 15.10 and 13) and the physiognomic note at the end of Valens' obituary. These sections are not entirely integrated into the narrative flow and add points inconsistent with the main arguments in the book XXXI. This helps better understand the historian's literary technique and some apparent inconsistencies in the last book of the Res Gestae.

Key words: Ammianus; book XXXI; sources, structure and composition of the Res Gestae; digressions; military narrative; intertextuality; physiognomy; character portrayal.

* Una versión previa de este trabajo fue presentada con el título «Loose Ends: On Some Stylistic Continuities in Ammianus' Book 31», en el coloquio «Ammianus Marcellinus: Past, Present, Future: A workshop at Ludwig Maximilians Universität», celebrado en Múnich los días 17 y 18 de junio de 2016. Agradezco los comentarios que me hicieron los asistentes y el relator del coloquio. Asimismo, agradezco las observaciones y sugerencias de los informantes de la revista. Todos ellos me han ayudado a perfilar los argumentos y a mejorar la calidad del escrito. Los errores e imprecisiones son, evidentemente, de mi exclusiva responsabilidad.

Copyright: (C) 2019 CSIC. Este es un artículo de acceso abierto distribuido bajo los términos de la licencia de uso y distribución Creative Commons Reconocimiento 4.0 Internacional (CC BY 4.0). 
Cómo citar este artículo / Citation: Sánchez-Ostiz, Álvaro 2019: «Fuentes y fases de redacción en el libro XXXI de Amiano Marcelino», Emerita 87 (2), pp. 317-339.

\section{Introducción}

A diferencia de los libros XIV-XXX que tratan alternativamente sucesos en el Este y el Oeste del Imperio ${ }^{1}$, el libro XXXI de las Res Gestae de Amiano Marcelino sigue un único hilo narrativo principal centrado en las acciones de Valente que prepara el clímax de la batalla de Adrianópolis y las consecuencias inmediatas de este desastre. Asimismo, en el último libro la persona narrativa del autor tiene una presencia menos marcada ${ }^{2}$, los exempla tienen una significación más analítica que moral $^{3}$ y las secciones digresivas son más escasas ${ }^{4}$.

Esta relativa discontinuidad del libro final de las Res Gestae ha sido objeto de diversos intentos de explicación ${ }^{5}$, desde Thompson ${ }^{6}$, que atribuye las asimetrías a la falta de habilidad literaria del autor, hasta $\mathrm{Sabbah}^{7}$, que reconoce las peculiaridades del libro XXX, pero insiste sin embargo en su coherencia con los libros XXVI-XXX ${ }^{8}$. Sin embargo, el mayor avance en la

${ }^{1}$ Siguiendo además en el caso de XIV-XXV una distribución en periodos sucesivos de verano e invierno: punto puesto de relieve ya por Seeck 1906 y tratado más claramente por Barnes 1998, pp. 32-42. Sobre la estructura dramática singular del libro XXXI incide asimismo Rosen 1970, pp. 186-189.

2 Den Boeft, Drijvers, den Hengst, Teitler 2018, pp. IX-XVI.

${ }^{3}$ Como destaca Blockley 1994, especialmente pp. 59-60.

${ }^{4}$ Solamente incluye el excurso sobre los hunos y alanos (XXXI 2), una observación sobre los desastres sufridos por Roma en el pasado (5.10-17) y la sphragis (16.9).

${ }^{5}$ Sobre estructura de las Res Gestae, ofrecen un panorama actualizado Matthews 1989, pp. 27-30; Sabbah 2003, pp. 47-50; Bocci 2013, pp. 21-25 y Harto Trujillo 2016.

${ }_{6}$ Thompson 1947, p. 121.

7 Sabbah 1997, pp. 107-114, Sabbah y Angliviel de la Beaumelle 1999, pp. VII-XVIII.

${ }_{8}$ Paso por alto que la numeración en 31 libros es incongruente con el hecho de que la obra esté estructurada en héxadas, tal como se deduce de las unidades que forman los libros XIV-XIX, XX-XXV y XXVI-XXXI. En su día, Michael 1880 supuso que Amiano habría combinado inicialmente dos obras, según el modelo taciteano de Historiae y Annales (véase la crítica de Jeep 1888). Para una discusión actualizada de la supuesta tradición taciteana de Amiano véase Kelly 2009. Para Barnes 1998, 20-31, el historiador habría compuesto una obra en 36 libros, 18 de ellos perdidos, pero un error en la transmisión manuscrita cambió el número del que habría sido el libro XIX por XIV, un desliz paleográficamente posible, pero difícil de demostrar. Posiblemente, la solución se halla en que, tal como sugiere Kelly 2008, 
comprensión del libro XXXI dentro del proyecto literario de Amiano se ha debido a Kulikowski 2012, quien propone que el último libro habría tenido como origen una obra de carácter monográfico según el modelo de los Skythika de Dexipo, escrita en griego ${ }^{9}$ por el propio historiador antes que los libros anteriores, en el contexto del debate intelectual surgido en Antioquía ${ }^{10}$ poco después del desastre del 9 de agosto del 378, durante los años 379-383, en los que Amiano habría estado en la ciudad siria, antes de trasladarse a Roma. En efecto, el libro XXXI de Amiano insiste precisamente en la responsabilidad de Valente en el desastre ${ }^{11}$, en los precedentes de la historia de Roma que superan con creces lo acontecido en Tracia ${ }^{12}$ y en que la masacre de godos reclutados en el ejército o asentados en territorio romano, llevada a cabo por Julio a finales del verano o comienzos de otoño del 378, fue salutaris et uelox para los intereses de Roma ${ }^{13}$. Estos contenidos parecen responder específicamente al discurso XXIV de Libanio (año 378-379) «Sobre la venganza por la muerte de Juliano», que atribuye la gran derrota a un castigo

p. 3, las Res Gestae comenzarían con un libro I que trataba a modo de epítome la historia de Roma desde la muerte de Nerva hasta Constantino, seguido de cinco héxadas.

${ }^{9}$ No es probable que una obra destinada a un público antioqueno se hubiese escrito en latín.

${ }^{10}$ Para un examen detallado de las reacciones al desastre de Adrianópolis en el Este y el Oeste del Imperio, véase Lenski 1997.

${ }^{11}$ Entre otros pasajes, XXXI 12.3: imperator procaci quodam calore perculsus isdem occurrere festinabat. Salvo mención expresa de lo contrario, se sigue el texto latino de la edición de Seyfarth 1978.

12 XXXI 5.11: Negant antiquitatum ignari tantis malorum tenebris offusam aliquando fuisse rem publicam, sed falluntur malorum recentium stupore confixi. namque si superiores uel recens praeteritae reuoluantur aetates, tales tamque tristes rerum motus saepe contigisse monstrabunt; XXXI 13.19: Constatque uix tertiam euasisse exercitus partem... annalibus praeter Cannensem pugnam ita ad internecionem res legitur gesta, quamquam Romani aliquotiens reflante Fortuna fallaciis lusi bellorum iniquitati cesserunt ad tempus et certamina multa fabulosae neniae fleuere Graecorum.

${ }_{13}$ XXXI 16.8: His diebus efficacia Iulii, magistri militiae, trans Taurum enituit salutaris et uelox. Comperta enim fatorum sorte per Thracias, Gothos antea susceptos dispersosque per uarias ciuitates et castra, datis tectioribus litteris ad eorum rectores, Romanos omnes, quod his temporibus raro contingit, uniuersos tamquam uexillo erecto, uno eodem die mandauit occidi, exspectatione promissi stipendii securos ad suburbana productos. Quo consilio prudenti sine strepitu uel mora completo, orientales prouinciae discriminibus ereptae sunt magnis. Sobre la fecha de la masacre, den Boeft, Drijvers, den Hengst, Teitler 2018, pp. 293-296. 
de los dioses por haber dejado sin sanción la muerte de Juliano ${ }^{14}$, y a los discursos XV y XVI de Temistio, que destacan las dimensiones nunca vistas de este suceso histórico ${ }^{15}$, así como el discurso XXIV, que defiende la conveniencia de la política de pactos con los godos que estaba llevando a cabo Teodosio ${ }^{16}$. En consecuencia, el historiador, después de terminar el libro XXX, habría completado su obra añadiendo una versión latina de su monografía anterior, agregando y retocando algunos puntos, dejando en el texto, sin embargo, señales notorias de falta de revisión.

En su opinión, la continuidad de rasgos de estilo y de técnica literaria presentes en los libros XIV-XXX en el último libro sólo podría probar, en estricta lógica, que Amiano escribió efectivamente el libro 31, pero no explicarían las pequeñas incongruencias dentro de la estructura del libro, de la última héxada y del conjunto de los libros conservados. Es decir, la presencia de una determinada coincidencia intertextual o de una auto-alusión en el libro final no permite inferir necesariamente que el libro 31 hubiese sido escrito después de los libros anteriores, como tampoco podría excluirse que hubiera sido añadido más tarde en un proceso de revisión que intentase armonizar el texto definitivo.

Sin duda, la hipótesis de Kulikowski presenta numerosos puntos sugerentes y aporta una explicación plausible de la especificidad del libro XXXI. En su exhaustiva discusión analiza en detalle numerosos pasajes del último libro y extrae indirectamente implicaciones que afectan a la cohesión interna de la última héxada y por extensión de toda la obra de Amiano. Si bien algunas de las interpretaciones concretas pueden ser discutibles o matizables, no se puede obviar que ha situado la parte final de las Res Gestae en una nueva perspectiva, abriendo una línea que permite seguir profundizando en la interpretación de la obra de Amiano. En consecuencia, aun aceptando el planteamiento general de que pudo haber un subtexto o un borrador primario, de género monográfico, escrito o no por Amiano Marcelino originalmente en griego, pueden plantearse otras hipótesis complementarias que expliquen puntos concretos de su propuesta: por ejemplo, que Amiano utilizase más de una fuente principal para componer el último libro; que esa auto-fuente no fuera el ori-

\footnotetext{
${ }^{14}$ Lib., Or. XXIV, especialmente 3-5 y 14-16.

15 Them., Or. XV, especialmente 190d, y XVI, especialmente 206d.

16 Them., Or. XXXIV, especialmente 22.
} 
gen de todo el libro sino tan solo de partes nucleares a las que habría añadido secciones; o que el paso del original griego a la versión latina no hubiera sido una traducción pegada a la letra sino una versión literaria que amplificaba sustancialmente el original. Complementariamente, podría resultar igualmente provechoso examinar si esas secciones añadidas tenían cierta extensión o constaban tan sólo de pequeñas apostillas o retoques.

El propósito de este trabajo no es discutir específicamente la existencia de una monografía escrita por Amiano, sino tomar como punto de partida que Amiano se sirvió como fuente principal para el libro XXXI de una monografía De Bello Gothico (a la que me referiré con la abreviatura DBG), para examinar si es posible detectar diferentes fases de redacción dentro del último libro de las Res Gestae, más concretamente, si se puede distinguir entre material original del DBG y material añadido por Amiano posteriormente. La valoración de los resultados de este examen permitirá a su vez establecer conclusiones sobre la peculiar estructura del libro XXXI, lo que podría redundar en un mejor conocimiento de la técnica literaria del historiador en pasajes de otros libros. Es preciso adelantar que algunas de las secciones del libro habrían sido añadidas con total seguridad, como es el caso del epílogo o de la transición de comienzo que enlaza con libros anteriores. Asimismo, se puede deducir que otras secciones se habrían hallado ya en el opúsculo original, porque muestran diferencias notorias con el modo de tratar escenas o contenidos similares en libros anteriores, o porque presentan una explicación o valoración de los acontecimientos en torno al desastre de Adrianópolis y la muerte de Valente, que no se habían presentado en libros anteriores. Por contraste, los argumentos para identificar que otras secciones habrían podido ser añadidas más adelante son diferentes en cada caso y no pueden atribuirse a un único rasgo de forma o contenido. Por esa razón, este estudio dedica los siguientes apartados a tres casos diferentes en los que el historiador podría haber acomodado secundariamente contenidos sobre el núcleo original: el excurso sobre los hunos y los alanos, las escenas bélicas estereotipadas que muestran interesantes diferencias dentro del mismo libro, y, por último, el obituario de Valente en el contexto del relato de su muerte.

\section{El excurso sobre los hunos y los alanos}

Amiano dedica el capítulo 2 del libro XXXI a un excurso etnográfico de cierta extensión sobre la apariencia y las costumbres de dos pueblos, los hunos (2-11) 
y los alanos (17-25), a los que atribuye ser la causa de la desgracia de Adrianópolis. Entre ambas descripciones hay una transición sobre los lugares que podrían haber ocupado los dos pueblos. Al tratarse de una sección que comparte evidentes puntos de contacto con otras descripciones etnográficas en las Res Gestae, la presencia de este excurso podría indicar una composición continuada del último libro. Kulikowski cuestiona este argumento, llamando la atención sobre las singularidades que la digresión de hunos y alanos tiene respecto a las incluidas en libros anteriores, en especial el que no incluya secciones sobre el contexto geográfico según el rígido patrón que ha utilizado en excursos anteriores ${ }^{17}$. Por otro lado, no es de excluir que la monografía DBG incluyera un excurso de este tipo, si se piensa en el modelo de Dexipo, que, en la medida en que se puede inferir de los fragmentos conservados, incluía descripciones de asedios y discursos, así como posiblemente digresiones ${ }^{18}$.

Sin embargo, esta misma particularidad puede deberse a que haya sido desligado intencionalmente de contexto geográfico, como es el caso del excurso sobre los sarracenos en XIV 14. La finalidad de esta digresión es poner de relieve la singularidad de dos pueblos, cuyos rasgos son estrictamente opuestos a los de civilización o «romanidad». En otras palabras, el excurso de hunos y alanos resulta diferente, al igual que el de los sarracenos, porque Amiano subraya que los pueblos descritos son en parte diferentes de los otros pueblos a los que se enfrentó Roma y presentan un tipo de amenaza nunca visto antes: su nomadismo.

El análisis de las fuentes y los contenidos en la presentación de los hunos muestra que el historiador ha combinado estereotipos del nomadismo con elementos fantasiosos, de modo que presenta una imagen literaria del «absolutamente distinto» que no persigue la precisión etnográfica ${ }^{19}$. Por esto mis-

${ }^{17}$ Kulikowski 2012, pp. 86-87.

${ }_{18}$ Martin 2006, pp. 161-162. El supuesto de que los Skythika de Dexipo habrían incluido excursos etnográficos se basa en FGrHist 30 (Fragm. 29 Martin), un pasaje de los Getica de Jordanes (XXII 113) que alude una descripción que Dexipo habría hecho de los asdingos: ... qui Asdingorum stirpe (quae inter eos eminet genusque indicat bellicosissimum, Dexippo historico referente, qui eos ab Oceano ad nostrum limitem uix in anni spatio peruenisse testatur prae nimia terrarum immensitate).

${ }_{19}$ Los análisis más exhaustivos sobre falta de rigor en la imagen construida por Amiano han sido los de Richter 1974 (que revisa las fuentes desde las que el historiador pudo reelaborar su descripción y la bibliografía anterior) y Vergin 2013, pp. 259-268 (que incide en la construcción de la «alteridad»o «anti-romanidad» que lleva a cabo Amiano en la digresión). 
mo, se puede descartar que el excurso esté basado en un improbable conocimiento directo de los hunos o alanos ${ }^{20}$. Sin embargo, Amiano sugiere en el lector que él ha tenido conocimiento exclusivo, privilegiado $\mathrm{y}$, por tanto, incomprobable, de aquello que describe. Precisamente por esta razón, Amiano parece implicar indirectamente lo contrario al afirmar que los hunos son tan rápidos en sus ataques que llegan a ser invisibles (XXXI 2.8): Vtque ad pernicitatem sunt leues et repentini, ita subito de industria dispersi rarescunt et incomposita acie, cum caede uasta discurrunt, nec inuadentes uallum nec castra inimica pilantes prae nimia rapiditate cernuntur. En este pasaje, un verbo de percepción visual en presente como cernuntur conecta la idea remota de los hunos con el momento mismo del lector; no obstante, resulta engañoso interpretar que esa inmediatez es consecuencia de la experiencia del autor y no un caso de «autopsia implícita» ${ }^{21}$. Es decir, no se puede desligar esta presentación de los pueblos nómadas de la reconocida tendencia del propio autor a sugerir una experiencia vívida y directa de lo narrado o de lo descrito para sugerir autoridad retórica a un punto de su relato que resulta incomprobable. Otros ejemplos de este mismo recurso en el libro XXXI, en los que Amiano acompaña el relato de intensidad realista y sensorial, apuntan igualmente a que la hipérbole sobre la rapidez de los hunos no depende de una vivencia del autor. Así, en XXXI 7.16 (... ut indicant nunc usque albentes ossibus campi), el historiador parece dar a entender que habría visitado tiempo antes el campo de batalla cerca de Marcianópolis ${ }^{22}$, pero el detallado análisis de $\mathrm{Kelly}^{23}$ muestra cómo la alusión a Virgilio y a Tácito ${ }^{24}$ esconde $^{2}$

Por su parte, Burgersdijk 2016, que centra su atención en la función literaria del excurso, acepta asimismo los términos generales de una elaboración más imaginativa que científica. Matthews 1989, pp. 332-342 no descarta un núcleo de información fidedigna.

${ }^{20} \mathrm{O}$ en lo que pudiera haber obtenido de testimonios orales oídos a informantes godos: sobre este supuesto, ya cuestionado por Richter 1974, pp. 364-365, véase la discusión en den Boeft, Drijvers, den Hengst, Teitler 2018, p. 11.

${ }^{21}$ Idea desarrollada por extenso en Kelly 2008, especialmente pp. 14-19.

${ }^{22}$ Cf., sin embargo, Sabbah 1978, p. 282 y Matthews 1989, pp. 16-17. Sobre el oppidum Salices y su dudosa identificación con el Ad Salices en la desembocadura del Danubio, a más de cien kilómetros de distancia de Marcianópolis, véase la discusión en den Boeft, Drijvers, den Hengst, Teitler 2018, pp. 111-113.

${ }^{23}$ Kelly 2008, pp. 13-30.

${ }^{24}$ Verg., Aen. XII 36: sanguine adhuc campique ingentes ossibus albent; Tac., Ann. I 61.2: media campi albentia ossa. 
sutilmente una polémica con la política teodosiana de acomodación de los godos después de Adrianópolis, puesto que nunc usque se ha de entender como un reproche a la situación de incuria a la que se había llegado, no a un improbable viaje del historiador. Igualmente, los desmanes que llevan a cabo los godos tras recuperarse del episodio anterior están descritos como si el autor y el lector fueran testigos oculares de esas acciones abominables en XXXI 8.7 (tunc erat spectare cum gemitu facta dictu uisuque praedira...); sin embargo, la acumulación de detalles sangrientos es tópica, tal como muestra el carácter estereotipado de la descripción que se detalla en el apartado siguiente. De manera similar, en XXXI 13.4 (... uidereque licebat celsum ferocia barbarum, genis stridore constrictis, succiso poplite aut abscisa ferro dextera uel confosso latere, inter ipsa quoque mortis confinia minaciter circumferentem oculos truces) Amiano agrega intensidad a un episodio concreto de la batalla de Adrianópolis, detallando cómo se podía percibir visualmente (uidere ... licebat) el daño infligido a los godos añadiendo así dramatismo a ese instante.

Desde este punto de vista, parece incongruente que las primeras líneas del excurso hagan mención tanto de un supuesto recurso a fuentes literarias anteriores, como de la escasez de referencias a los hunos en testimonios antiguos (XXXI 2.1):

Totius autem sementem exitii et cladum originem dinersarum, quas Martius furor incendio insolito miscendo cuncta conciuit, hanc comperimus causam. Hunorum gens monumentis ueteribus leuiter nota ultra paludes Maeoticas glacialem oceanum accolens omnem modum feritatis excedit.

Por un lado, Amiano parece aludir con hanc comperimus causam ${ }^{25}$ que ha basado su excurso en fuentes literarias: de hecho, se nota la presencia de Heródoto en partes de su descripción ${ }^{26}$. En esta línea, no sería ilógico que Amiano hubiese tomado su descripción fantasiosa de fuentes escritas anteriores, desconocidas para la posteridad, y que se haya limitado a trasladar ese material derivativo, sin que haya quedado posibilidad de comprobarlo. Por

${ }^{25}$ Amiano utiliza comperio sistemáticamente para referirse a 'recabar información': den Boeft, Drijvers, den Hengst, Teitler 2018, p. 13 ad loc.; véase especialmente XXV 10.13 y XXXI 13.17.

${ }^{26}$ Matthews 1989, pp. 334-335. 
otro lado, si el pueblo huno es apenas mencionado en testimonios anteriores (Hunorum gens monumentis ueteribus leuiter nota), el objeto de ese comentario parece más bien sugerir indirectamente que su descripción se basa en un conocimiento directo que, como ya se ha mencionado, no resulta creíble. La analogía en este punto con el recurso que Amiano hace en otros puntos a la «autopsia implícita» para revestirse de un conocimiento superior o autorizado sobre la materia en cuestión ${ }^{27}$, permite suponer que, en el caso de esta particular colección de curiosidades sobre hunos y alanos, quiere infundir autoridad a sus contenidos más incomprobables. Dos argumentos apuntan a que Amiano no sólo modela imaginativamente el excurso, sino que además lo incluye en una segunda fase de redacción. En primer término, existe una importante incongruencia entre la justificación del excurso basada en que los hunos han sido la causa de todas las calamidades (totius autem sementem exitii et cladum originem diuersarum) y el papel más bien modesto, casi testimonial y anecdótico, que este pueblo tiene en el relato del resto del libro, apareciendo esporádicamente en 3.1, 3, 6, 8.4 y 16.328. En segundo término, la función literaria que tiene en el relato es precisamente de cohesión temática y estructural con el relato anterior, sirviendo de punto de inflexión para introducir seguidamente la narrativa bélica que conduce a Adrianópolis ${ }^{29}$. En definitiva, el excurso de los hunos y los alanos es un excurso con características peculiares, pero no esencialmente diferente de los precedentes en XIVXXX. Es más, resulta paradójico que el excurso insista en que hunos y alanos son enemigos completamente distintos, por ser esencialmente «anti-civilizados», cuando el punto de vista predominantemente subrayado en el libro XXXI y específicamente acentuado en 5.10-17 es que el desastre de Adrianópolis no fue un suceso sin precedentes. Por último, las peculiaridades, como la ausencia de fórmulas de digresión y regreso al relato o la inusual colocación de los contenidos geográficos, pueden considerarse precisamente signos de una acomodación apresurada de este material secundario en el contenido principal del libro, tomado del DBG.

27 Kelly 2008, pp. 63-65.

${ }_{28}$ Kulikowski 2012, pp. 86-87; Burgersdijk 2016, p. 120.

${ }_{29}$ Para Burgersdijk 2016, que analiza en detalle la función literaria de esta sección, el propósito de Amiano es acentuar que la gran amenaza de Roma es lo opuesto a la civilización y marcar la transición hacia el relato del desastre militar propiamente dicho. 


\section{Viñetas estereotipadas en la narrativa militar}

Por el propio carácter del libro XXXI, en el que la preparación del desastre de Adrianópolis centra el hilo conductor del relato, las descripciones de acciones bélicas dominan el relato salvo en los capítulos 1, 2, 5.10-17, 14 y 16.9. Es importante subrayar que en estas secciones de narrativa militar el autor de las Res Gestae utiliza los mismos recursos estilísticos y de composición que en los libros anteriores, en especial las expresiones estereotipadas que no proporcionan información específica sobre el desarrollo concreto de una acción y contrastan con secciones sin elementos estereotipados que sí transmiten información específica ${ }^{30}$. A su vez, algunas expresiones vagas del relato pueden estar combinadas con unidades narrativas que sí aportan información precisa y relevante para el momento descrito.

En estricta lógica, tanto si las presentaciones mediante lenguaje estereotipado en el libro XXXI reflejan la realidad de los sucesos de manera verosí$\mathrm{mil}^{31}$, como si son constructos secundarios de secciones para las que Amiano no disponía de información ${ }^{32}$, la presencia de ese recurso no implica necesariamente que el libro XXXI fuera escrito realmente a continuación de los demás y no mucho antes, puesto que las coincidencias podrían haber surgido más tarde en el proceso de acomodación del material del último libro. En esta misma línea, tampoco parece metodológicamente posible precisar si una determinada fórmula en el libro XXXI estaba ya en el hipotético DBG e influye, una vez vertida al latín, sobre la redacción de libros anteriores, o bien surge en el proceso de redacción de los libros XIV-XXX y Amiano la utiliza para cohesionar el texto que acaba de incorporar al final de las Res Gestae.

Sin embargo, sí se puede establecer que los pasajes en los que se tratan escenas típicas, pero no se detectan fórmulas descriptivas, sino información

${ }^{30}$ Bitter 1976 establece los diferentes patrones que sigue el autor y desarrolla categorías de clasificación tanto de escenas típicas de contienda como de motivos y clichés recurrentes.

${ }_{31}$ Matthews 1989, pp. 286-301 (especialmente p. 295) y Kelly 2008, pp. 63-65 cuestionan que el mero uso de clichés sea necesariamente indicio de que el relato es inventado, destacando que las acciones que Amiano describe de manera formular podrían haber tenido lugar de hecho, si no exactamente, sí de manera similar a como describe Amiano. En esta misma línea, Crump 1975, especialmente pp. 91-96, subraya que la exageración estilística de Amiano en su narrativa de batallas y asedios reduce la información a lo dramático y esencial, pero ello no implica que inventara el relato por completo.

32 Bitter 1976, pp. 191-195. 
específica, responden a material original que Amiano no ha revisado ni nivelado estilísticamente. Este es el caso de XXXI 13, en el que Amiano describe una multitud compacta en su narración de la batalla de Adrianópolis de manera ciertamente diferente a como lo hace más adelante en dos ocasiones al presentar el asedio de la ciudad en el capítulo 15. A priori, dado que el relato de la batalla es el clímax que van preparando los capítulos anteriores, es de suponer que el capítulo XXXI 13 estuviera ya en el DBG en griego, si no en su totalidad, sí a grandes rasgos. Es de notar que las líneas iniciales son inespecíficas y utilizan fórmulas que ya han aparecido en ocasiones anteriores para presentar el intenso intercambio entre los dos bandos de una batalla ${ }^{33}$. Por contraste, la información proporcionada por Amiano a continuación sobre la muchedumbre tan apretada, que se ve incapaz de movimiento y resulta blanco seguro de los disparos indiscriminados, es más específica y prescinde de alusiones como las que muestran secciones anteriores:

steterunt improtecti pedites ita concateruatis manipulis, ut uix mucronem exserere aut manus reducere quisquam posset. nec iam obiectu pulueris caelum patere potuit ad prospectum, clamoribus resultans horrificis. qua causa tela undique mortem uibrantia destinata cadebant et noxia, quod nec prouideri poterant nec caueri.

En este caso, los proyectiles arrojados por doquier en medio de la polvareda resultaban necesariamente mortíferos (destinata cadebant et noxia), puesto que sus destinatarios no podían verlos ni evitarlos, precisamente porque la masa estaba estrechamente compactada. La misma idea es retomada asimismo más adelante en el mismo libro XXXI, en dos pasajes distintos dentro del capítulo 15, durante el asedio de Adrianópolis después de la gran derrota. En el primero, Amiano menciona cómo era imposible que los defensores romanos erraran sus disparos, incluso sin apuntar (uel temere missa) ante la apretada multitud de enemigos (XXXI 15.10): ... et cuiusce modi tela in multitudine tanta, uel temere missa, cadere sine noxa non poterant, mientras que en el segundo es la codicia y el deseo de emulación lo que provoca

33 13.1: Cumque arma ex latere omni concuterentur et tela lituosque Bellona luctuosos inflaret in clades Romanas, solito immanius furens, cedentes nostri multis interclamantibus restiterunt, et proelium flammarum ritu accrescens, terrebat militum animos confixis quibusdam rotatis ictibus iaculorum et sagittarum. 
que los godos compacten las primeras líneas (XXXI 15.13): ... nullo ferme alio telo uel funditoris amento, in cassum excusso. Agmina enim praeeuntium ductorum, quos rapiendi Valentis malis lucrationibus quaesita cupiditas incendebat, secuti, ceteri prae se ferebant aequiperasse discrimina potiorum. La diferencia entre el motivo tal como es usado en el capítulo 13 y en el 15 estriba en que, en el segundo caso, la expresión sigue un patrón común con otros pasajes similares en libros anteriores, por ejemplo, en XIX 2.13 (moxque clamore sublato cunctis temere prorumpentibus telorum uis ingens uolabat e muris, utque opinari dabatur, nulla frustra mittebantur inter hominum cadentia densitatem) o en XX 7.6 (... sagittarum enim nimbi crebrius uolitantes stantes confertius perforabant $)^{34}$.

Por consiguiente, se puede suponer por una parte que la materia tratada por Amiano en XXXI 13, que es el núcleo del libro XXXI, se hallaba en el original del DBG, mientras que el modo estereotipado de tratar una situación similar en el capítulo 15 sugiere que éste ha sido añadido y modelado más tarde en una fase de redacción diferente. En ambos casos, sin embargo, el propósito del historiador al subrayar la estrecha cohesión y densidad de las tropas enemigas es destacar la superioridad numérica del contingente godo en aquellos eventos de los que Valente era en último término responsable: la batalla y en el asedio de Adrianópolis. Es probable, por tanto, que Amiano haya expandido aquellas secciones que le permiten intensificar el contraste de las acciones de Valente con las de los emperadores anteriores, un punto de vista que resultaría añadido al integrar la monografía DBG en el conjunto de las Res Gestae.

No obstante, es necesario precisar que no toda información específica en el contexto de un relato bélico con profusión de fórmulas ha de proceder necesariamente del DBG, como corrobora el patrón descriptivo recurrente en

\footnotetext{
${ }^{34}$ Cuya expresión depende a su vez de un motivo tradicional en la literatura latina y fácilmente reconocible por el lector, como atestiguan pasajes de Verg., Aen. XI 610-611 (fundunt simul undique tela / crebra niuis ritu caelumque obtexitur umbra); Liu. XXXVIII 26.7 (nec procurrere quisquam ab ordinibus suis, ne nudarent undique corpus ad ictus, audebant, et stantes quo densiores erant hoc plura, uelut destinatum petentibus, uolnera accipiebant); XXX 18.10 (in quos cum pila confertos coniecissent nullo ferme frustra emisso); Sen., Cons. Marc. XVI 5 (nullum aiunt frustra cadere telum, quod in confertum agmen inmissum est). Otra expresión que concurre sin ser específica del motivo de la multitud densamente apretada es clamore undique sublato, imitada probablemente de Livio (véase Liu. II 10.9; XXXVII 32.11; XXXIX 35.8).
} 
libros anteriores y bien conocido por la tradición historiográfica, que Amiano reutiliza en dos pasajes del libro XXXI (6.7-8 y 8.7-8) para describir las devastadoras correrías de los godos en Tracia. Este patrón está basado, principal pero no exclusivamente, en tres motivos estereotipados: una multitud inocente sufre acciones atroces por parte de los vencedores; éstos no hacen distinción de edad o sexo entre sus víctimas; y éstas acaban siendo arrastradas como si fueran un rebaño de animales ${ }^{35}$.

En el caso de XXXI 6, turbas de rebeldes godos, después de unirse a Fritigerno en Adrianópolis, vagan por Tracia y con la ayuda de desertores devastan el territorio, masacrando a sus habitantes, sin distinción de sexo o edad (sine distantia enim aetatis uel sexus); los niños pequeños son arrancados de los brazos de sus madres y asesinados, las viudas pasadas a cuchillo, y los jóvenes arrastrados en medio de los cadáveres de sus padres, mientras los ancianos se quejan del cruel giro del destino (XXXI 6.7-8):

Sine distantia enim aetatis uel sexus caedibus incendiorumque magnitudine cuncta flagrabant abstractisque ab ipso uberum suctu paruulis et necatis raptae sunt matres et uiduatae maritis coniuges ante oculos caesis et puberes adultique pueri per parentum cadauera tracti sunt. senes denique multi ad satietatem uixisse clamantes post amissas opes cum speciosis feminis manibus post terga contortis defletisque gentilium fauillis aedium ducebantur extorres.

Por su parte, algunos elementos de contenido y de expresión de este pasaje son recogidos a su vez en XXXI 8.7-8, que presenta un contexto parecido, pero al que Amiano añade nuevos matices ${ }^{36}$ :

Tunc erat spectare cum gemitu facta dictu uisuque praedira, attonitas metu feminas flagris concrepantibus agitari, fetibus grauidas adhuc immaturis, antequam prodirent in lucem, impia tolerantibus multa: implicatos alios matribus paruulos; et puberum audire lamenta puellarumque nobilium, quarum stringe-

${ }^{35}$ Bitter 1976, p. 53, n. 159. Den Boeft, Drijvers, den Hengst, Teitler 2018, pp. 148-149 lo denominan de manera genérica urbs capta topos y remiten a los precedentes de la literatura latina tratados por Paul 1982.

${ }^{36}$ Como señalan Sabbah 1978, p. 543, n. 8, subrayando la función persuasiva que el patetismo del pasaje tiene sobre el lector, y Sabbah y Angliviel de la Beaumelle 1999, p. 264, n. 442. 
bat fera captiuitas manus. post quae adulta uirginitas castitasque nuptarum ore abiecto flens ultima ducebatur mox profanandum pudorem optans morte licet cruciabili praeuenire. inter quae cum beluae ritu traheretur ingenuus paulo ante diues et liber, de te Fortuna ut inclementi querebatur et caeca, quae eum puncto temporis breui opibus exutum et dulcedine caritatum domoque extorrem, quam concidisse uidit in cinerem et ruinas, aut lacerandum membratim aut seruiturum sub uerberibus et tormentis crudo deuouisti uictori.

En este caso, no se menciona la ausencia de discrimen sexus uel aetatis, sino que el historiador sustituye la explicación por una enumeración de feminas, grauidas, paruuli, matres, puberes, puellae acompañada de una expresión colectiva adulta uirginitas castitasque, que es paralela a los paruuli, matres, coniuges, puberes, pueri y senes mencionados en XXXI 6.7-8. Por contraste, XXXI 8.7-8 acentúa más la presentación vívida a través de una fórmula de «autopsia implicada» tunc erat spectare y la percepción sensorial del terror sufrido por las víctimas: ... impia tolerantibus multa ... attonitas metu ... audire lamenta ... ore abiecto flens...

En esta misma línea de inmediatez sugerida indirectamente, la viñeta narrativa del hombre rico que es arrastrado como si de una bestia se tratase (beluae ritu traheretur), mientras lloriquea lamentándose de la despiadada Fortuna, parece propiamente contenido específico y concreto, pero sin duda está insertada para intensificar el momento dramático, por lo que ha de considerarse un añadido de la imaginación de Amiano ${ }^{37}$. Otros paralelos léxicos, como extorres / extorrem, amissas opes / opibus exutum o tracti sunt / traheretur señalan asimismo la interdependencia de ambos pasajes por alusión o, más probablemente, por compartir un mismo patrón presente ya en la tradición historiográfica latina ${ }^{38} \mathrm{y}$ en contextos similares de los libros XIV-XXX: XVI 11.9 (un comando de auxiliares con el tribuno Bainobaudes lleva a cabo un estrago entre los alamanes) ${ }^{39}$; XVII 13.12 (Constancio vence y expulsa los limigantes) ${ }^{40}$; XIX 9.2 (destino de los generales romanos capturados en

\footnotetext{
${ }^{37}$ Kulikowski 2012, p. 93.

38 Véase, por ejemplo, Liu. I 8.6; VI 27.7; XXI 15.1; XXVIII 3.10.

${ }^{39}$ Egressique promisce uirile et muliebre secus sine aetatis ullo discrimine trucidabant ut pecudes.

40 Vixdum populis hostilibus stratis gregatim peremptorum necessitudines ducebantur humilibus extractae tuguriis, aetatis sexusque promiscui et fastu uitae prioris abolito ad
} 
Amida) ${ }^{41}$; XX 6.7 (Sapor conquista Singara y expulsa a sus habitantes) ${ }^{42}$; XX 7.15 (Sapor conquista Bezabde) ${ }^{43}$; XXIV 4.25-26 (los romanos toman la ciudad de Maiozamalcha) ${ }^{44}$ y XXIX 6.8 (los cuados devastan Panonia y Valeria $)^{45}$.

Estrictamente no se puede establecer con seguridad si Amiano compuso estas secciones de manera progresiva o regresiva, es decir, si las escenas de urbs capta y, por extensión, de muchedumbre llevada indiscriminadamente hacia la esclavitud en XXXI 6.7-8 y 8.7-8 son una amplificación modelada sobre escenas semejantes en libros anteriores o una acomodación de última hora de material original, o bien si aquéllas ya estaban en su monografía DBG desde una fase preliminar, de modo que las escenas semejantes en los libros XIV-XXX las toman como punto de referencia. Es necesario destacar, no obstante, que Kulikowski detectó dos llamativas ausencias de referencia cruzada en XXXI 8: la mención de Saturnino en 8.3 que ignora su mención anterior en XXII 3.7 y la de Barzimeres en 8.9, presentado como si apareciera por primera vez, a pesar de haber sido nombrado antes en XXX 1.1146, por lo que es probable que al menos el contenido general de la sección estuviera ya en la monografía DBG. Sin embargo, en ambos pasajes, en el capítulo 6 y 8 , Amiano ha adaptado un motivo conocido a un contexto inusual, trasladando el tópico de las consecuencias de un asedio o de una batalla a las

infimitatem obsequiorum uenere seruilium et exiguo temporis interuallo decurso caesorum aggeres et captiuorum agmina.

${ }^{41}$ Aliique protectores post terga uinctis manibus ducebantur Transtigritanis, qui sollicita quaerebantur industria, nullo infimi summique discrimine ad unum omnibus contruncatis. Por contraste, en XIX 8.4 Amiano utiliza el motivo para describir los estragos sufridos por ambos bandos en el desenlace del sitio de Amida: pecorum ritu armati et inbelles sine sexus discrimine truncabantur.

${ }^{42}$ Persarum agmina undique ululabili clamore sublato nullo cohibente cuncta oppidi membra complebant caesisque promisce paucissimis residui omnes mandatu Saporis uiui comprehensi ad regiones Persidis ultimas sunt asportati.

${ }^{43}$ Abreptique sinibus matrum paruuli ipsaeque matres trucidabantur nullo, quid ageret, respectante.

${ }^{44}$ Inuaditur ciuitas et sine sexus discrimine uel aetatis, quidquid impetus repperit, potestas iratorum absumpsit.

${ }^{45}$ Praedas hominum uirile et muliebre secus agebant et pecorum uillarum cineribus exustarum caesorumque incolentium exsultantes aerumnis, quos necopinantes sine ulla parsimonia deleuerunt.

${ }^{46}$ Kulikowski 2012, p. 89, n. 57. 
razias de los godos en Tracia. Éstos, al igual que los hunos y alanos del excurso analizado anteriormente, son un enemigo sin domicilio que, amparado en sus carromatos, representa una amenaza diferente para Roma. Se podría considerar, por tanto, que ambos pasajes XXXI 6.7-8 y 8.7-8 pudieron ser un inserto posterior para cohesionar el relato con la idea del enemigo nómada.

\section{El obituario de Valente}

Estrechamente unidos con las dos versiones sobre la muerte de Valente, el obituario del emperador y la referencia al oráculo de Mimas completan el núcleo central del libro XXXI. La necrología repasa las uirtutes y los uitia del Emperador recién fallecido y tiene como punto de referencia evidente de las notas necrológicas similares que Amiano ha insertado sobre los demás emperadores que han protagonizado su relato: tres más extensas, sobre Constancio (XXI 16), Juliano (XXV 4), y Valentiniano (XXX 7-9) y otras tres más breves, sobre Galo (XIV 11.27-29), Joviano (XXV 10.14-15) y Procopio (XXVI 9.11). La de Valente muestra en efecto puntos de conexión con las otras seis, que siguen un patrón bien definido ${ }^{47}$, pero se diferencia de éstas al menos en dos aspectos dignos de mención ${ }^{48}$. Por una parte, esta necrología es la más breve con diferencia en comparación con las tres más extensas, lo que podría deberse tanto a que habría sido escrita antes que el resto en el DBG, como a que fue incorporada más adelante como una expansión del material. Por otra parte, las buenas cualidades de Valente (2-4) aparecen inesperadamente, puesto que no han sido detalladas antes en el relato, y son tratadas antes de lo uitia (4-6), en orden inverso al resto. Esta estructura no permite en principio dilucidar si Amiano ha incorporado en este punto material de otra fuente, para equilibrar la nota final y acomodarla a lo que había hecho en los casos anteriores, o si el obituario del capítulo XXXI 14 pertenecía al material derivado de DBG. Sin embargo, tres argumentos permiten inferir que el obituario, en su totalidad o en varias de sus partes, ha sido añadido más tarde a manera de arreglo o acomodo del relato a motivos que habían sido tratados ya en los libros XIV-XXX.

${ }_{47}$ Pauw 1977, pp. 186-188; Sabbah 1978, pp. 449-450; Matthews 1989, pp. 239-242.

${ }^{48}$ Kelly 2008, p. 265; Den Boeft, Drijvers, den Hengst, Teitler 2018, pp. 243-244. 
En primer término, los términos utilizados en el comentario sobre la falta de educación literaria de Valente ${ }^{49}$ (XXXI 14.5: subagrestis ingenii, nec bellicis nec liberalibus studiis eruditus) continúan comentarios de pasajes anteriores que tratan con mayor extensión y detalle ese defecto del emperador, recogiendo la misma expresión (XXIX 1.11: subrusticum hominem; XXX 4.2: subagreste ingenium nullius uetustatis lectionibus expolitum). En caso de este pasaje del libro XXX, la observación sobre la rudeza de Valente está hecha en el contexto de las acciones de Modesto, que aparta al emperador del ejercicio de la justicia. Precisamente a continuación, el excurso sobre los abogados de Antioquía en XXX 4.8-22 desarrolla por extenso la decadencia de la educación oratoria como causa de los males del Imperio ${ }^{50}$, en directa alusión al contraste con Juliano, por lo que es más probable que el comentario final haya sido escrito con posterioridad.

En segundo término, el obituario se cierra con la nota fisonómica de Valente en los párrafos 7-8, que presenta el mismo patrón seguido rigurosamente por Amiano en el caso de Constancio ${ }^{51}$, Juliano ${ }^{52}$ y Valentiniano ${ }^{53}$, más concretamente: la constitución corporal, el color del pelo, la mirada y la manera de caminar:

7 Cessator et piger, nigri coloris, pupula oculi unius obstructa, sed ita, ut non eminus appareret, figura bene compacta membrorum, staturae nec procerae nec humilis, incuruis cruribus exstanteque mediocriter uentre. 8 Haec super Valente dixisse sufficiet, quae uera esse aequalis nobis memoria plene testatur.

49 Den Boeft, Drijvers, den Hengst, Teitler 2018, p. 250 y Lenski, 2002, pp. 94-95.

${ }^{50}$ Sánchez-Ostiz 2016.

${ }^{51}$ XXI 16.19: Figura tali situque membrorum: subniger luce oculorum edita cernensque acutum, molli capillo, rasis assidue genis lucentibus ad decorem, ad usque pubem ab ipsis colli confiniis longior, breuissimis cruribus et incuruis, unde saltu ualebat et cursu.

${ }^{52}$ XXV 4.22: Figura tali situque membrorum: mediocris erat staturae, capillis tamquam pexis et mollibus, hirsuta barba in acutum desinente uestitus, uenustate oculorum micantium flagrans, qui mentis eius argutias indicabant, superciliis decoris et naso rectissimo, ore paulo maiore, labro inferiore demisso, opima et incurua ceruice, umeris uastis et latis, ab ipso capite usque unguium summitates liniamentorum recta compage, unde uiribus ualebat et cursu.

${ }_{53}$ XXX 9.6: Corpus eius lacertosum et ualidum, capilli fulgor colorisque nitor, cum oculis caesiis, semper obliquum intuentis et toruum, atque pulchritudo staturae liniamentorumque recta compago maiestatis regiae decus implebat. 
Si no existiera el contexto de las demás notas fisonómicas, se podría entender que la inclusión de este contenido sobre Valente estaba dictada meramente por la convención retórica, según la tendencia del momento ${ }^{54}$, y resultaba natural en el DBG. Sin embargo, se ha de tener en cuenta que otros rasgos destacados en el XXXI 14, en especial, la ignorancia de Valente, ponen de relieve que Amiano ha querido establecer deliberadamente un contraste de Valente con Juliano, cuya inteligencia precisamente, según afirma la nota fisonómica correspondiente, se reflejaba en la mirada XXV 4.22: uenustate oculorum micantium flagrans, qui mentis eius argutias indicabant ${ }^{55}$. Por tanto, resulta probable que también la nota sobre la apariencia física haya sido añadida en XXXI 14, para reenfocar el final de Valente desde el punto de vista de la comparación con Juliano ${ }^{56} \mathrm{y}$, por ello, con los demás emperadores de las Res Gestae. Dos detalles sobre la autoridad que el historiador da a su propio comentario merecen atención. Por un lado, Amiano parece sugerir que habría visto personalmente el orzuelo de Valente cuando subraya que éste solo se advertía si se miraba de cerca ${ }^{57}$. Por otro, la apostilla final del obituario, que funciona prácticamente como una fórmula de regreso al final de un excurso (haec super Valente dixisse sufficiet, quae uera esse aequalis nobis memoria plene testatur), podría estar sugiriendo que Amiano tenía un recuerdo personal y directo de Valente como el que tenían sus contemporáneos $^{58}$. Sin embargo, tal como se ha mencionado acerca del excurso sobre los hunos y alanos, la insistencia en el conocimiento directo de un suceso o materia por parte de Amiano no es un argumento incontestable para establecer de hecho que ese fuera el caso. Sin duda, estas dos notas añaden autoridad

\footnotetext{
${ }^{54}$ Sobre la influencia en Amiano de las teorías fisonómicas en boga en su tiempo, véase, sobre todo, Sabbah 1978, pp. 421-428; Rohrbacher 2010, pp. 103-109 y Castillo 2016.

${ }^{55}$ Gregorio Nazianceno hace la interpretación opuesta de la mirada de Juliano, al que

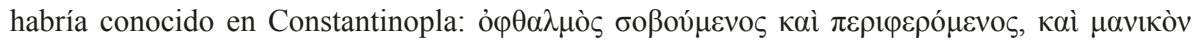
$\beta \lambda \dot{\varepsilon} \pi \omega v$ (Greg. Naz., Or. V 23).

${ }^{56}$ En esta línea, sobre la comparación implícita con Juliano y Estrasburgo en el relato de Adrianópolis, véase el análisis de Brodka 2009, pp. 119-126.

${ }^{57}$ Así lo interpreta Rohrbacher 2010, p. 108, en el contexto de la relación entre las Res Gestae y los tratados fisonómicos del momento.

${ }^{58}$ Lo que no resulta imposible puesto que ambos, Amiano y Valente, podrían haber coincidido entre los protectores domestici; no obstante, no hay certeza sobre la vinculación que Valente pudo tener con esa unidad: véase la discusión de las fuentes en Lenski 2002, pp. 51-52 y Kelly 2008, p. 130.
} 
retórica a una descripción que de otro modo resultaría fantasiosa y poco digna de crédito. Como apunta Rohrbacher ${ }^{59}$, la tradición fisonómica interpretaba que un orzuelo era signo del destino fatal e inminente de su portador, lo que a su vez muestra que Amiano ha utilizado la nota sobre la apariencia física de Valente para aludir indirectamente a la incapacidad de éste para captar el significado de los presagios sobre su destino inminente.

Por último, en tercer término, la necrología se completa en los párrafos 8-9 con una nueva referencia al oráculo mencionado en XXIX 1.33, que había profetizado la muerte del emperador en las llanuras de Mimas ( $\dot{\varepsilon} v$

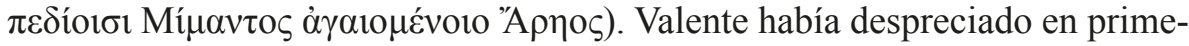
ra instancia la profecía (ut erat inconsummatus et rudis, inter initia contemnebat), pero más tarde inquiere sobre su significado. Los expertos le informan de que el oráculo se refiere a un lugar cercano a la ciudad jonia de Eritras, por lo que el emperador evita acercarse a Asia Menor. Sin embargo, el oráculo se acaba cumpliendo en las llanuras de Tracia, pues un túmulo cercano al lugar en el que muere el emperador pertenecía a un noble de nombre Mimas: lapis affixus, incisis litteris Graecis, sepultum ibi nobilem quendam Mimanta ueterem indicabat. Como discute Kulikowski ${ }^{60}$ de manera detenida y convincente, esta referencia cruzada está insertada a posteriori para conectar artificial y superficialmente este pasaje del libro XXXI con el resto de la héxada final. A su argumento se puede añadir precisamente que la nueva referencia al oráculo, inmediatamente después de la mención del orzuelo con sus propias implicaciones premonitorias de la muerte del emperador, introduce de manera forzada una cuestión que no había aparecido antes en este libro: la capacidad que pudo tener Valente de entender e interpretar los signos sobre su destino. Aunque el primer capítulo del libro enumera los presagios y portentos que apuntaban a la derrota de Adrianópolis y al fin del emperador ${ }^{61}$, sin embargo, no menciona cuál pudo ser la reacción de éste

${ }^{59}$ Rohrbacher 2010, p 108.

${ }^{60}$ Kulikowski 2012, pp. 85-86. Para Bleckmann 2007, que discute en detalle el posible origen del relato sobre el oráculo, Amiano habría trabajado en la última héxada, y en especial en el último libro, refundiendo fuentes secundarias y no tanto sobre la base de informaciones de primera mano.

${ }^{61}$ De los que sólo uno, mencionado además superficialmente, es un signo celeste (XXXI 1.2: squalidi solis exortus hebetabant matutinos diei candores), a diferencia de los signos que presagian la muerte de Constancio o Joviano, que son mayoritariamente de orden astronómico. 
ante las advertencias que le podían proporcionar los expertos (1.2: quae uates auguresque praedixere ueridice). Por contraste, Amiano trata con profusión de detalle e incluso un excurso científico las reacciones de Constancio ante el eclipse (XX 3.2-12) y el arco iris (20.11.25-30), de Juliano ante la aparición del genius publicus (XXV 2.4-6) y de Joviano ante la visión de un cometa (XXV 10.2-3). Por tanto, la perspectiva de la incapacidad de Valente para interpretar signos, que es un aspecto específico de su ignorancia, ha estado ausente hasta que es rescatada en el final del obituario, en lo que con toda probabilidad es un añadido posterior que une de manera artificiosa las presentaciones imperiales anteriores con la de Valente para armonizar el conjunto.

\section{Conclusiones}

Una vez discutidos los diversos argumentos, se puede afirmar que al menos los tres supuestos descritos no se hallaban en la primera fase de redacción del libro XXXI, sino que fueron añadidos por Amiano en una fase posterior: la digresión sobre los hunos y los alanos en XXXI 2, desarrollada con abundancia de detalles fantasiosos y dotada de la autoridad erudita impostada; algunas secciones breves dentro de la narrativa del libro, concretamente XXXI 6.7-8, $8.7-8,15.10$ y 13 , que no proporcionan información específica y muestran coincidencias cercanas de expresión con otras descripciones contenidas en libros anteriores; y la nota fisonómica al final del obituario de Valente en XXXI 14.7-8, que sigue el mismo patrón que las notas similares a propósito de Constancio, Juliano y Valentiniano. Los tres casos no están perfectamente integrados en el conjunto del libro XXXI desde un punto de vista formal, por falta de cohesión interna en el caso del excurso etnográfico, por no añadir información relevante en la narración bélica o por incoherencia entre los hechos de Valente y su necrología, al tiempo que aluden a contenidos que trascienden los límites del último libro y lo cohesionan con el resto de las Res Gestae.

Este análisis no ha cuestionado la tesis planteada por Kulikowski de una monografía escrita por el propio Amiano, sino que se ha servido de sus intuiciones para avanzar en el análisis de la singularidad del libro XXXI. La hipótesis de una obra escrita en griego, en un género inusual en el momento y como respuesta intelectual a las reacciones antioquenas al desastre de Adrianópolis, insertada y acomodada más tarde en la trama a modo de epílogo por 
el propio autor, no puede evitar que algunos de sus elementos sean más especulativos que otros. No obstante, esta conjetura ayuda ciertamente a comprender mejor el papel estructural del libro XXXI y el mérito literario de la obra historiográfica más ambiciosa de la literatura latina tardía.

Por último, si bien se han podido detectar signos de distintas fases de redacción en el libro XXXI, se ha puesto de relieve asimismo que el autor utilizó las fases subsiguientes para integrar el final dentro de un todo al que, de hecho, da sentido como proyecto literario. Los relativos desequilibrios y discontinuidades en la estructura de las Res Gestae, las que afectan al libro XXXI y las que afectan a los anteriores, no obstan para percibir que Amiano tenía en mente desde el principio de su gran obra un contraste entre Estrasburgo y Adrianópolis, y un contraste entre Juliano, Constancio, Valentiniano y Valente. Por lo tanto, a pesar de que pudiera haberse servido de una fuente principal anterior, compuesta o no por el mismo autor, el libro XXXI fue desde el comienzo una parte esencial de su proyecto literario para el conjunto de las Res Gestae, en el que pasajes de los libros anteriores anticipan pasajes del último y se entienden mejor teniendo en cuenta el conjunto.

\section{BiBLIOGRAFÍA}

Barnes, T. D. 1998: Ammianus Marcellinus and the Representation of Historical Reality, Ithaca.

Bitter, N. 1976: Kampfschilderungen bei Ammianus Marcellinus, Bonn.

Bleckmann, B. 2007: «Vom Tsunami von 365 zum Mimas-Orakel: Ammianus Marcellinus als Zeithistoriker und die spätgriechische Tradition», en den Boeft, J., den Hengst, D. y Teitler, H. C. (eds.), Ammianus after Julian: the Reign of Valentinian and Valens in Books 26-31 of the Res Gestae, Leiden, pp. 7-31.

Blockley, R. C. 1994: «Ammianus Marcellinus's Use of Exempla», Florilegium 13, pp. 53-64.

Bocci, S. 2013: Ammiano marcellino, XXVIII e XXIX: Problemi storici e storiografici, Roma.

Den Boeft, J., Drijvers, J. W., den Hengst, D. y Teitler, H. C. 2008: Philological and Historical Commentary on Ammianus Marcellinus XXVI, Leiden.

Den Boeft, J., Drijvers, J. W., den Hengst, D. y Teitler, H. C. 2018: Philological and Historical Commentary on Ammianus Marcellinus XXXI, Leiden.

Brodka, D. 2009: Ammianus Marcellinus: Studien zum Geschichtsdenken im vierten Jahrhundert n. Chr., Cracovia. 
Burgersdijk, D. 2016: «Creating the Enemy: Ammianus Marcellinus’ Double Digression on Huns and Alans (Res Gestae 31.2)», BICS 59, pp. 111-132.

Castillo, C. 2016: «Historia, physiognomia y encomio: la figura de Graciano en Amiano Marcelino», en Sánchez-Ostiz, Á. (ed.), Beginning and End: From Ammianus Marcellinus to Eusebius of Caesarea, Huelva, pp. 159-173.

Crump, G. A. 1975: Ammianus Marcellinus as a Military Historian, Wiesbaden.

Harto Trujillo, M. L. 2016: «Amiano Marcelino y las Res Gestae», Emerita 84 (1), pp. 121-144.

Jeep, L. 1888: «Die verlorenen Bücher des Ammianus», RhM 43, pp. 60-72.

Kelly, G. 2007: «The sphragis and Closure of the Res Gestae», en den Boeft, J., Drijvers, J. W., den Hengst, D. y Teitler, H. C. (eds.), Ammianus after Julian: The Reign of Valentinian and Valens in Books 26-31 of the Res Gestae, Leiden, pp. 219-241.

Kelly, G. 2008: Ammianus Marcellinus: The Allusive Historian, Cambridge.

Kelly, G. 2009: «Ammianus Marcellinus: Tacitus' Heir and Gibbon's Guide», en Feldherr, A. (ed.), The Cambridge Companion to the Roman Historians, Cambridge, pp. 348-361.

Kulikowski, M. 2012: «Coded Polemic in Ammianus Book 31 and the Date and Place of Its Composition», JRS 102, pp. 79-102.

Lenski, N. 1997: «Initium mali Romano imperio: Contemporary Reactions to the Battle of Adrianople», TAPhA 127, pp. 129-168.

Lenski, N. 2002: Failure of Empire: Valens and the Roman State in the Fourth Century AD, Berkeley.

Martin, G. 2006: Dexipp von Athen: Edition, Übersetzung und begleitende Studien, Tubinga.

Matthews, J. 1989: The Roman Empire of Ammianus, Londres.

Michael, H. 1880: Die verlorenen Bücher des Ammianus Marcellinus: Ein Beitrag zur römischen Literaturgeschichte, Breslavia.

Paul, G. M. 1982: «Urbs Capta: Sketch of an Ancient Literary Motif», Phoenix 36, pp. 144-155.

Pauw, D. A. 1977: «Methods of Character Portrayal in Ammianus Marcellinus», AClass 20, pp. 181-198.

Richter, W. 1974: «Die Darstellung der Hunnen bei Ammianus Marcellinus (31,2,111)», Historia 23, pp. 343-377.

Rohrbacher, D. 2010: «Physiognomics in Imperial Latin Biography», ClAnt 29, pp. 92-116.

Rosen, K. 1970: Studien zur Darstellungskunst und Glaubwürdigkeit des Ammianus Marcellinus, Bonn.

Sabbah, G. 1978: La méthode d'Ammien Marcellin: Recherches sur la construction du discours historique dans les Res Gestae, París. 
Sabbah, G. 1997: «Ammien Marcellin, Libanius, Antioche et la date des derniers livres des Res gestae», Cassiodorus 3, pp. 89-116.

Sabbah, G. 2003: «Ammianus Marcellinus», en Marasco, G. (ed.), Greek and Roman Historiography in Late Antiquity: Fourth to Sixth Century AD, Leiden, pp. 43-84.

Sabbah, G. y Angliviel de la Beaumelle, L. 1999: Ammien Marcellin. Histoire, VI, livres $X X I X-X X X I$, París.

Sánchez-Ostiz, Á. 2016: «Ammianus on Eastern Lawyers (30.4): Literary Allusions and the Decline of Forensic Oratory», en Sánchez-Ostiz, Á. (ed.), Beginning and End: From Ammianus Marcellinus to Eusebius of Caesarea, Huelva, pp. 207-223.

Seeck, O. 1906: «Zur Chronologie und Quellenkritik des Ammianus Marcellinus», Hermes 4, pp. 481-539.

Seyfarth, W. 1978: Ammiani Marcellini Rerum gestarum libri qui supersunt, Leipzig. Thompson, E. A. 1947: The Historical Work of Ammianus Marcellinus, Cambridge. Vergin, W. 2013: Das Imperium Romanum und seine Gegenwelten: Die geographisch-ethnographischen Exkurse in den Res Gestae des Ammianus Marcellinus, Berlín. 